Int. Journal of Math. Analysis, Vol. 7, 2013, no. 43, 2129 - 2136

HIKARI Ltd, www.m-hikari.com

http://dx.doi.org/10.12988/ijma.2013.36138

\title{
Distribution of the Zeros of the Generalized Euler Polynomials of Second Kind
}

\author{
C. S. Ryoo \\ Department of Mathematics \\ Hannam University, Daejeon 306-791, Korea \\ Y. H. Kim \\ Department of Mathematics \\ Hannam University, Daejeon 306-791, Korea \\ H. Y. Jung \\ Department of Mathematics \\ Hannam University, Daejeon 306-791, Korea
}

Copyright (C) 2013 C. S. Ryoo et al. This is an open access article distributed under the Creative Commons Attribution License, which permits unrestricted use, distribution, and reproduction in any medium, provided the original work is properly cited.

\begin{abstract}
In this paper, we observe the behavior of complex roots of the generalized Euler polynomials $\widetilde{\mathcal{E}}_{n}(x)$ of the second kind, using numerical investigation. By means of numerical experiments, we demonstrate a remarkably regular structure of the complex roots of the generalized Euler polynomials $\widetilde{\mathcal{E}}_{n}(x)$ of the second kind. Finally, we give a table for the solutions of the generalized Euler polynomials $\widetilde{\mathcal{E}}_{n}(x)$ of the second kind.
\end{abstract}

Mathematics Subject Classification: 11B68, 11S40, 11S80

Keywords: Euler numbers and polynomials, generalized Euler polynomials of the second kind, complex roots, numerical experiments 


\section{Introduction}

Recently, many authors have studied the Euler numbers and polynomials, Euler numbers and polynomials of the second kind. The Euler numbers and polynomials, Euler numbers and polynomials the second kind possess many interesting properties and arising in many areas of mathematics and physics. We introduce the the generalized Euler polynomials $\widetilde{\mathcal{E}}_{n}(x)$ of the second kind. In order to study the generalized Euler polynomials $\widetilde{\mathcal{E}}_{n}(x)$ of the second kind, we must understand the structure of the generalized Euler polynomials $\widetilde{\mathcal{E}}_{n}(x)$ of the second kind. Therefore, using computer, a realistic study for the generalized Euler polynomials $\widetilde{\mathcal{E}}_{n}(x)$ of the second kind is very interesting.

It is the aim of this paper to observe an interesting phenomenon of 'scattering' of the zeros of the generalized Euler polynomials $\widetilde{\mathcal{E}}_{n}(x)$ of the second kind in complex plane. The outline of this paper is as follows. In Section 2, introduce the generalized Euler polynomials $\widetilde{\mathcal{E}}_{n}(x)$ of the second kind. In Section 3 , we describe the beautiful zeros of the generalized Euler polynomials $\widetilde{\mathcal{E}}_{n}(x)$ of the second kind using a numerical investigation. Finally, we investigate the roots of the generalized Euler polynomials $\widetilde{\mathcal{E}}_{n}(x)$ of the second kind. Also we carried out computer experiments for doing demonstrate a remarkably regular structure of the complex roots of the generalized Euler polynomials $\widetilde{\mathcal{E}}_{n}(x)$ of the second kind.

\section{Generalized Euler polynomials $\widetilde{\mathcal{E}}_{n}(x)$}

In this section, we define the generalized Euler numbers and polynomials of the second kind. First, we introduce the Genocchi numbers and Genocchi polynomials. The Genocchi numbers $G_{n}$ are defined by the generating function:

$$
\frac{2 t}{e^{t}+1}=\sum_{n=0}^{\infty} G_{n} \frac{t^{n}}{n !}, \quad(|t|<\pi)
$$

where we use the technique method notation by replacing $G^{n}$ by $G_{n}(n \geq 0)$ symbolically. The second kind Euler numbers $\widetilde{\mathcal{E}}_{n}$ are defined by the generating function(see [4]):

$$
\left(\frac{2 e^{t}}{e^{2 t}+1}\right)=\sum_{n=0}^{\infty} \widetilde{\mathcal{E}}_{n} \frac{t^{n}}{n !}, \quad\left(|t|<\frac{\pi}{2}\right)
$$

In [4], we introduced the second kind Euler numbers $\widetilde{\mathcal{E}}_{n}$ and investigate their properties. First of all, we define the generalized Euler polynomials $\widetilde{\mathcal{E}}_{n}(x)$ of the second kind as follows. 
Definition 2.1 Let $x$ be a real or complex parameter. The generalized Euler polynomials $\widetilde{\mathcal{E}}_{n}(x)$ of the second kind are defined by means of the generating function:

$$
\left(\frac{2 e^{t}}{e^{2 t}+1}\right)^{x}=\sum_{n=0}^{\infty} \widetilde{\mathcal{E}}_{n}(x) \frac{t^{n}}{n !}\left(|t|<\frac{\pi}{2} ; 1^{x}:=1\right),
$$

Setting $x=1$ in (2.2), we can obtain the corresponding definition for the second kind Euler numbers.

By (2.1) and Definition 2.1, we easily have

$$
\widetilde{\mathcal{E}}_{n}(1)=\sum_{k=0}^{n}\left(\begin{array}{l}
n \\
k
\end{array}\right) G_{k+1} \frac{2^{k}}{k+1}
$$

As well known definition, the Stirling number $s(n, k)$ of the first kind can be defined by means of

$$
(x)_{n}=x(x-1) \cdots(x-n+1)=\sum_{k=0}^{n} s(n, k) x^{k} .
$$

Stirling number $S(n, k)$ of the second kind can be defined by

$$
x^{n}=\sum_{k=0}^{n} S(n, k) \frac{x^{n}}{n !} .
$$

We obtain relationship between the Stirling numbers and the generalized Euler polynomials of second kind. By Definition 2.1, (2.4), (2.5), we have the following theorem.

Theorem 2.2 For $n \geq k(n, k \in \mathbb{N})$, we have

$$
\widetilde{\mathcal{E}}_{n}(x)=\sum_{k=0}^{n} c(n, k) x^{k},
$$

where $c(n, k)=\sum_{j=0}^{k}\left(\begin{array}{c}n \\ j\end{array}\right)(-1)^{k-j} \sum_{l=k-j}^{n-j} s(l, k-j) S(n-j, l) 2^{n-j-l}$.

Setting $n=1,2,3,4,5,6,7,8,9,10$ in Theorem 2.2, we get to

$$
\begin{aligned}
& \widetilde{\mathcal{E}}_{0}(x)=1, \quad \widetilde{\mathcal{E}}_{1}(x)=0, \\
& \widetilde{\mathcal{E}}_{2}(x)=-x, \quad \widetilde{\mathcal{E}}_{3}(x)=0, \\
& \widetilde{\mathcal{E}}_{4}(x)=2 x+3 x^{2}, \quad \widetilde{\mathcal{E}}_{5}(x)=0, \\
& \widetilde{\mathcal{E}}_{6}(x)=-16 x-30 x^{2}-15 x^{3}, \quad \widetilde{\mathcal{E}}_{7}(x)=0, \\
& \widetilde{\mathcal{E}}_{8}(x)=272 x+588 x^{2}+420 x^{3}+105 x^{4}, \quad \widetilde{\mathcal{E}}_{9}(x)=0, \\
& \widetilde{\mathcal{E}}_{10}(x)=-7936 x-18960 x^{2}-16380 x^{3}-6300 x^{4}-945 x^{5},
\end{aligned}
$$


Remark 2.3 Let $n, m \in \mathbb{N}$. Then by Theorem 2.2, we have

$$
\sum_{k=0}^{n} c(n, k)=\sum_{k=0}^{n}\left(\begin{array}{l}
n \\
k
\end{array}\right) G_{k+1} \frac{2^{k}}{k+1} .
$$

From Remark 2.3, we easily see that $\sum_{k=0}^{n} c(n, k) x^{k}$ generalized Euler polynomials is related to Genocchi numbers.

By applying Theorem 2.2, we have

$$
k ! c(n, k)=\left.\frac{d^{k}}{d x^{k}} \widetilde{\mathcal{E}}_{n}(x)\right|_{x=0}
$$

On the other hand, it follows from (2.2) that

$$
\begin{gathered}
\left(\log \frac{2 e^{t}}{e^{2 t}+1}\right)^{k}=\sum_{n=0}^{\infty} \frac{d^{k}}{d x^{k}}\left(\left.\widetilde{\mathcal{E}}_{n}(x)\right|_{x=0}\right) \frac{t^{n}}{n !} \\
k ! \sum_{n=k}^{\infty} c(n, k) \frac{t^{n}}{n !}=\left(\log \frac{2 e^{t}}{e^{2 t}+1}\right)^{k}
\end{gathered}
$$

Now note

$$
\frac{d}{d t} \log \left(\frac{2 e^{t}}{e^{2 t}+1}\right)=\sum_{n=0}^{\infty} G_{n+1} \frac{2^{n}}{n+1} \frac{t^{n}}{n !}-1 .
$$

By integrating from 0 to $t$ in(2.8), we deduce that

$$
\log \frac{2 e^{t}}{e^{2 t}+1}=\sum_{n=2}^{\infty} \frac{2^{n-1} G_{n}}{n} \frac{t^{n}}{n !}
$$

Substituting (2.9) in (2.7) we get

$$
c(n, k)=\frac{2^{n-k} n !}{k !} \sum_{\substack{v_{1}+\cdots+v_{k}=n \\ 1 \leq i \leq k, 2 \leq v_{i}}} \frac{G v_{1} \cdots G v_{k}}{\left(v_{1} \cdots v_{k}\right)\left(v_{1} ! \cdots v_{k} !\right)} .
$$

Therefore, we have the following theorem.

Theorem 2.4 For $n \geq k(n, k \in \mathbb{N})$, we have

$$
\begin{aligned}
& \sum_{\substack{v_{1}+\\
+v_{k}=n \\
1 \leq i \leq k, 2 \leq v_{i}}} \frac{G v_{1} \cdots G v_{k}}{\left(v_{1} \cdots v_{k}\right)\left(v_{1} ! \cdots v_{k} !\right)} \\
& =\frac{k !}{n !} \sum_{j=0}^{k-1}\left(\begin{array}{c}
n \\
j
\end{array}\right)(-1)^{k-j} \sum_{l=k-j}^{n-j} s(l, k-j) S(n-j, l) 2^{k-j-l} .
\end{aligned}
$$

Remark 2.5 Setting $k=1$ in Theorem 2.4, and noting that $s(j, 1)=$ $(-1)^{j-1}(j-1)$ !, we obtain

$$
G_{n}=2 n \sum_{l=1}^{n} \frac{(-1)^{l}(l-1) !}{2^{l}} S(n, l)(n \geq 2) .
$$




\section{Zeros of the generalized Euler polynomials $\widetilde{\mathcal{E}}_{n}(x)$ of the second kind}

In this section, we investigate the reflection symmetry of the zeros of the generalized Euler polynomials $\widetilde{\mathcal{E}}_{n}(x)$ of the second kind. Since

$$
\begin{aligned}
\sum_{n=0}^{\infty} \widetilde{\mathcal{E}}_{n}(x) \frac{(-t)^{n}}{n !} & =\left(\frac{2 e^{-t}}{e^{-2 t}+1}\right)^{x} \\
& =\left(\frac{2 e^{t}}{e^{2 t}+1}\right)^{x} \\
& =\sum_{n=0}^{\infty} \widetilde{\mathcal{E}}_{n}(x) \frac{t^{n}}{n !}
\end{aligned}
$$

we have $\left.\widetilde{\mathcal{E}}_{n}(x)=(-1)^{n} \widetilde{\mathcal{E}}_{n}(x)\right)$ for $n \in \mathbb{N}$.

Hence, we arrive at the following theorem.

Theorem 3.1 For $n \in \mathbb{N}$ and $n \equiv 1(\bmod 2)$, we have

$$
\widetilde{\mathcal{E}}_{n}(x)=0
$$

Throughout the numerical experiments, we can finally conclude that $\widetilde{\mathcal{E}}_{n}(x), x \in$ $\mathbb{C}$ has not $\operatorname{Re}(x)=0$ reflection symmetry analytic complex functions. However, we observe that $\widetilde{\mathcal{E}}_{n}(x), x \in \mathbb{C}$ has $\operatorname{Im}(x)=0$ reflection symmetry(see Figure 1). The obvious corollary is that the zeros of $\widetilde{\mathcal{E}}_{n}(x)$ will also inherit these symmetries.

$$
\text { If } \widetilde{\mathcal{E}}_{n}\left(x_{0}\right)=0 \text {, then } \widetilde{\mathcal{E}}_{n}\left(x_{0}^{*}\right)=0 \text {, }
$$

where $*$ denotes complex conjugation(see Figure 1 and Figure 3).

We investigate the beautiful zeros of the generalized Euler polynomials $\widetilde{\mathcal{E}}_{n}(x)$ by using a computer. We plot the zeros of the generalized Euler polynomials $\widetilde{\mathcal{E}}_{n}(x)$ for $n=20,30,40,50$ and $x \in \mathbb{C}$ (Figure 1 ). In Figure 1 (top-left), we choose $n=20$. In Figure 1(top-right), we choose $n=30$. In Figure 1(bottomleft), we choose $n=40$. In Figure 1(bottom-right), we choose $n=50$.

Plots of real zeros of $\widetilde{\mathcal{E}}_{n}(x)$ for $1 \leq n \leq 60$ structure are presented(Figure 2).

Stacks of zeros of $\widetilde{\mathcal{E}}_{n}(x)$ for $1 \leq n \leq 60$ from a 3 -D structure are presented(Figure 3). Our numerical results for approximate solutions of real zeros of the generalized $\widetilde{\mathcal{E}}_{n}(x)$ are displayed(Tables 1,2$)$. 

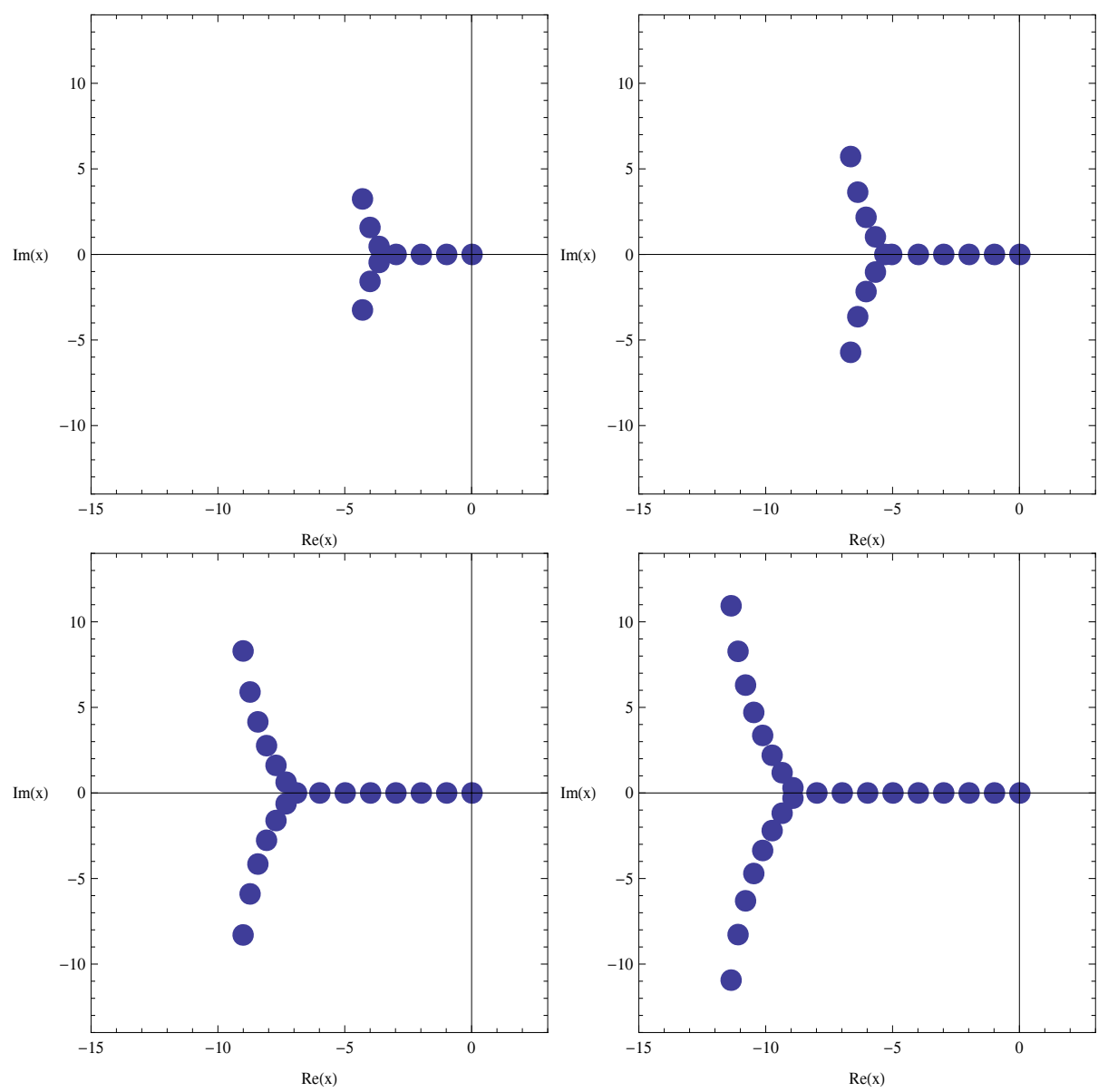

Figure 1: Zeros of $\widetilde{\mathcal{E}}_{n}(x)$ for $n=20,30,40,50$

Table 1. Numbers of real and complex zeros of $\widetilde{\mathcal{E}}_{n}(x)$

\begin{tabular}{|c||cc|}
\hline$n$ & real zeros & complex zeros \\
\hline \hline 2 & 1 & 0 \\
\hline 4 & 2 & 0 \\
\hline 6 & 1 & 2 \\
\hline 8 & 2 & 2 \\
\hline 10 & 3 & 2 \\
\hline 12 & 2 & 4 \\
\hline 14 & 3 & 4 \\
\hline 16 & 4 & 4 \\
\hline 18 & 3 & 6 \\
\hline 20 & 4 & 6 \\
\hline
\end{tabular}

We observe a remarkably regular structure of the complex roots of the gener- 


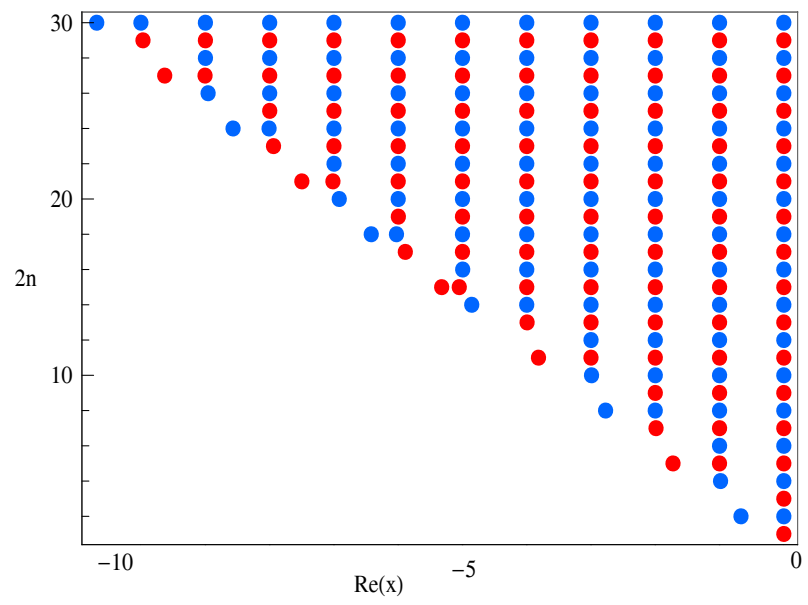

Figure 2: Real zeros of $\widetilde{\mathcal{E}}_{n}(x)$ for $1 \leq n \leq 60$

alized Euler polynomials $\widetilde{\mathcal{E}}_{n}(x)$. We hope to verify a remarkably regular structure of the complex roots of the generalized Euler polynomials $\widetilde{\mathcal{E}}_{n}(x)$ (Table $1)$. Next, we calculated an approximate solution satisfying $\widetilde{\mathcal{E}}_{n}(x), x \in \mathbb{R}$. The results are given in Table 2 .

Table 2. Approximate solutions of $\widetilde{\mathcal{E}}_{n}(x)=0, x \in \mathbb{R}$

\begin{tabular}{|l||c|}
\hline$n$ & $x$ \\
\hline \hline 2 & 0 \\
\hline 4 & $-0.6666666667,0$ \\
\hline 6 & 0 \\
\hline 8 & $-0.9845319214,0$ \\
\hline 10 & $-1.724316306, \quad-1.000758949,0$ \\
\hline 12 & $-1.988461938, \quad-1.000000377,0$ \\
\hline 14 & $-2.000783019, \quad-0.999999995, \quad 0$ \\
\hline 16 & $-2.773327957, \quad-1.000000000,0$ \\
\hline 18 & $-1.999963994, \quad-2.0$ \\
\hline 20 & $-2.992197642, \quad-2.000001294, \quad-1.00000000, \quad 0$ \\
\hline
\end{tabular}

Finally, we shall consider the more general problems. For $n \in \mathbb{N}, \widetilde{\mathcal{E}}_{2 n}(x)=$ 0 has $n$ distinct solutions. Find the numbers of complex zeros $C_{\widetilde{\mathcal{E}}_{2 n}(x)}$ of 


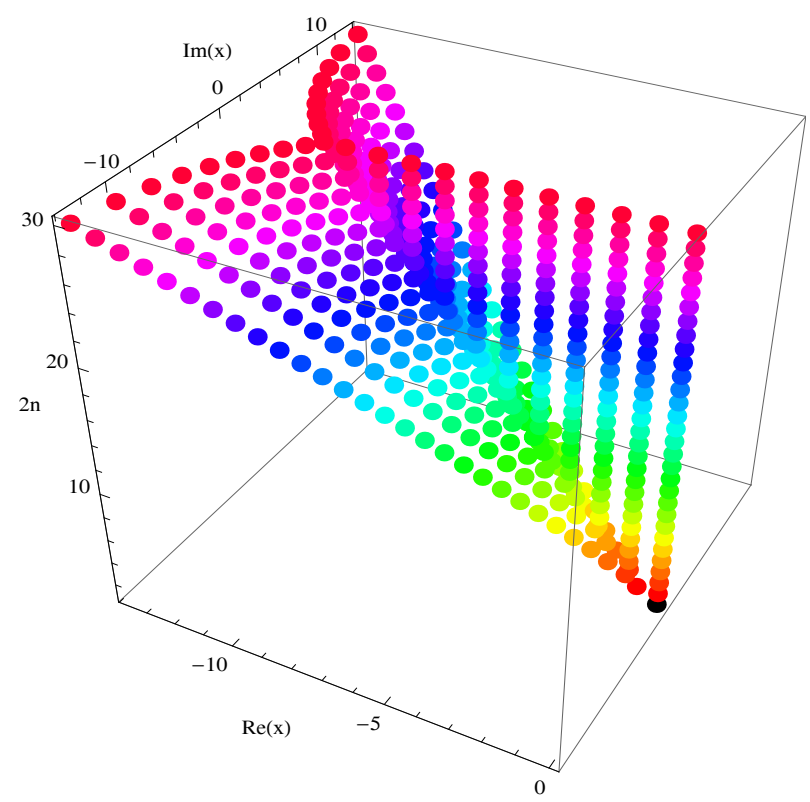

Figure 3: Stacks of zeros of $\widetilde{\mathcal{E}}_{n}(x)$

$\widetilde{\mathcal{E}}_{2 n}(x), \operatorname{Im}(x) \neq 0$. Since $n$ is the degree of the polynomial $\widetilde{\mathcal{E}}_{2 n}(x)$, the number of real zeros $R_{\widetilde{\mathcal{E}}_{2 n}(x)}$ lying on the real plane $\operatorname{Im}(x)=0$ is then $R_{\widetilde{\mathcal{E}}_{2 n}(x)}=$ $n-C_{\widetilde{\mathcal{E}}_{2 n}(x)}$, where $C_{\widetilde{\mathcal{E}}_{2 n}(x)}$ denotes complex zeros. See Table 1 for tabulated values of $R_{\widetilde{\mathcal{E}}_{2 n}(x)}$ and $C_{\widetilde{\mathcal{E}}_{2 n}(x)}$.

\section{References}

[1] R. Ayoub, Euler and zeta function, Amer. Math. Monthly, 81(1974), 1067-1086.

[2] T. Kim, Note on the Euler numbers and polynomials, Adv. Stud. Contemp. Math., 17(2008), 131-136

[3] C. S. Ryoo, A numerical computation on the structure of the roots of $q$ extension of Genocchi polynomials, Appl. Math. Lett., 21 (2008), 348-354.

[4] C. S. Ryoo, Calculating zeros of the second kind Euler polynomials, J. Comput. Anal. Appl., 12 (2010), 828-833.

[5] C. S. Ryoo, On the generalized twisted q-Euler numbers and polynomials with weak weight $\alpha$, Adv. Studies Theor. Phys., 7(2013), 245 - 251

Received: June 9, 2013 